\title{
Innovative Coating Materials for Glass Structures
}

\author{
František Novotný, Lenka Prokopová and Daniela Bošová
}

\begin{abstract}
As innovative materials based on glass our research picks substances that can be applied on the glass or glazed structures and objects and effects positively and improve the quality of their inner environment. In this research project is new material applied to the sample glass material and other matrices for which we assume "inappropriate" physical properties of thermal conductivity and light transmittance. Glass bubbles are used as thin-layered internal isolation.

Observed parameters are the thickness, color, structure and texture of applied materials. Among the major measurable values is light and heat transmission in both summer and winter (ie bidirectional passages of the heat and light energy). These parameters also relate to the requirements for the quality and quantity of throughput light and heat radiation.
\end{abstract}

Index Terms-Glass bubbles, hollow glass microsphere, coating material, isolation, thermal and daylight internal environment.

\section{INTRODUCTION}

For the first phase of the research is the decisive criterion suitability and feasibility of applying technology of the investigated material and its chosen carrier and adhesive medium, which vary depending on the used matrix and positioning applications (interior / exterior). Another point will be the following research limits of aesthetics and visual conception of glass applications - the choice of materials and means of expression. We will target the originality of the design, its practical application and contribution to the building industry.

\section{AIM}

The first phase of the project is the creation of prototypes and samples facade components with programmed heat and technical characteristics. We are looking for a state where the sample will have an optimum thickness of the glass (matrix) and coat, colour, texture and finish. All described endpoints are significantly influenced by application technology and used type of adhesive. The result will be functional glass components that meet the needs and demands of the proposal-required thermal and technical parameters.

Manuscript received March 19, 2017; revised July 12, 2017. This work was supported by the Czech Technical University in Prague - Student Grant Competition under Grant SGS15/222/OHK1/3T/15.

F. Novotný and D. Bošová. are with the Czech Technical University Institute of Building Technologies II, Prague 6, 16000 CZ (e-mail: novotfr2@fa.cvut.cz,daniela.bosova@fa.cvut.cz).

L. Prokopová was with the Czech Technical University Institute of Building Technologies II, Prague 6, 16000 CZ (e-mail: prokopova.lenka@centrum.cz).

\section{PROBLEM}

Innovative materials, which can be applied to the glass and glass structures, allow us to improve their physical properties and thus directly affect the quality of the internal environment inside the glazed objects. For us it is examined innovative material is again glass - its form - elements that exhibit extreme reflective properties and allows a significant improvement in thermal and technical properties of glass constructions.

We use simple monocellular hollow spherical elements, known as the "Glass Bubbles". They are made of borosilicate glass which is resistant to water and is chemically stable.

At a time when we are considering the reflective element, such as a very small particle, the glass begins to behave like a "disembodied" reflector or radiator. At this point for us is the layer of material a radiation blocker and in the figurative sense it behaves as an insulator. The actual insulating ability is not very big, but due to the high proportion of air / gas in a closed unventilated space between each tessellation of glass microsphere it has proven positive effect on improving thermal properties.

A major impact on thermal and technical characteristics of the applied material layer has an adhesive medium. Its percentage in the layer and the extent of surface covering the effective surface of the reflector, substantially affects the reflective capability of the layer.

\section{RESEARCH QUESTION}

\section{A. Main Research Question}

It is possible to use innovatively the material "Glass Bubbles" - 3M ${ }^{\mathrm{TM}}$ in order to improve thermal, technical and optical parameters of building structures and confirm that by empirical testing?

\section{B. Research Question for the First Phase}

What should be the proportion of binder in the coating layer and what chemical characteristics should it have to act as a reliable adhesive layer in terms of the exterior and interior, while has the smallest possible negative effect on the reflective ability of applied material?

\section{Description of the Methods / Used Techniques}

This research project is based on innovative application of known materials glass samples, glass models and samples of other thin building materials that are suspected to have inappropriate thermal properties. Examined parameters are the thickness, color, structure and texture of applied materials in relation to the quality of the inner environment. These are mainly thermal comfort in summer and winter. These 
parameters are monitored by measuring inner/affected light and temperature conditions, and depending on the results adjusted input parameters to achieve an optimal value, that meets the requirements for the quality and quantity of daylight and temperature inside the building.

Another endpoint are the limits of aesthetics and visual concept of the glass objects covered with functional layers. Selecting the studied materials and means of expression we target the originality of the design, its practical application and contribution to the industry of building glass.

\section{INTERIM RESULTS - DISCUSSION}

\section{A. Medium}

In the context of recent research of the problem was carried out search of existing methods of applying and fixing the investigated material and was chosen the most suitable method of application. This issue was discussed with the manufacturers and converters of studied material as well as authors of scientific papers on Faculty of Biomedical Engineering of Czech technical University in Prague (FBME CTU) who deals with the theme of adhesion of small particles of coating materials on optical fibres.

FBME CTU methods were found to be not feasible in terms of research at Faculty of Architecture at CTU.

The methods used by the material convertor - AZ TECH Ltd. arising from 3M - were complying with the size and funding of research and are further developed and adapted to respond according to the research.

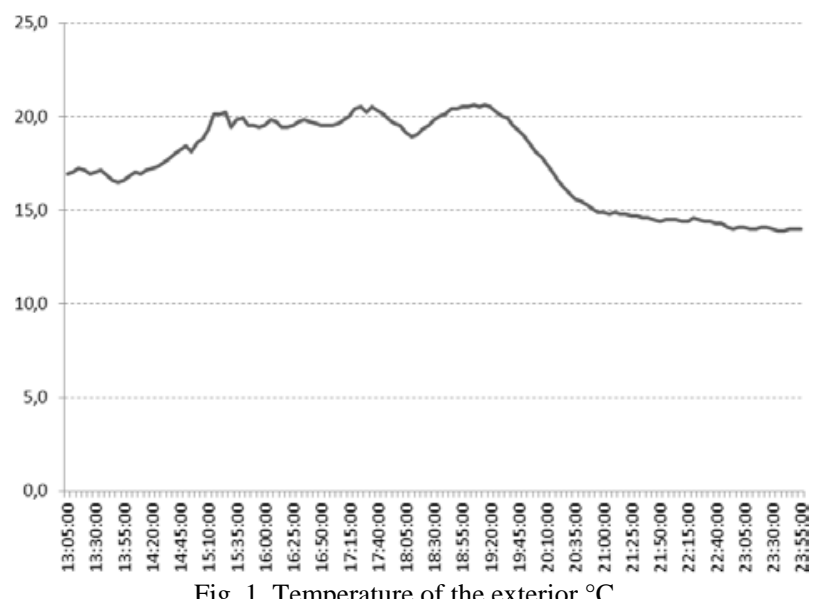

\section{B. Application}

We have conducted several experimental methods of application of the material. Selected method is application by using the directional "manual" spraying with the use of coating masks to achieve a programmed percentage coverage matrix. It was tested by the method of spraying machine, which has better controllability in thickness and consistency layer, but was found to be inappropriate and inflexible for the first stage of research.

\section{Thermal Properties}

Now there are on-going experimental measurements of thermal-technical parameters of the material on two fronts: 1 . calibrated samples of glass matrix with different surface proportions of the reflecting layer are tested in terms of penetration of solar radiation threw applied layer between interior and exterior. 2. Cargo container modules are tested in terms of heat gains and losses while one metal structure is with and second is without the reflective layer.

Measurement results are not yet to be published and serve as a model for finding substantial and insubstantial boundary conditions for measurements and calculations.

\section{Field Experiment}

By the time of ongoing field experiment - measurement of thermal-technical parameters of the material in terms of penetration of solar radiation through applied layer between interior and exterior on cargo container modules - we've come to first field results.
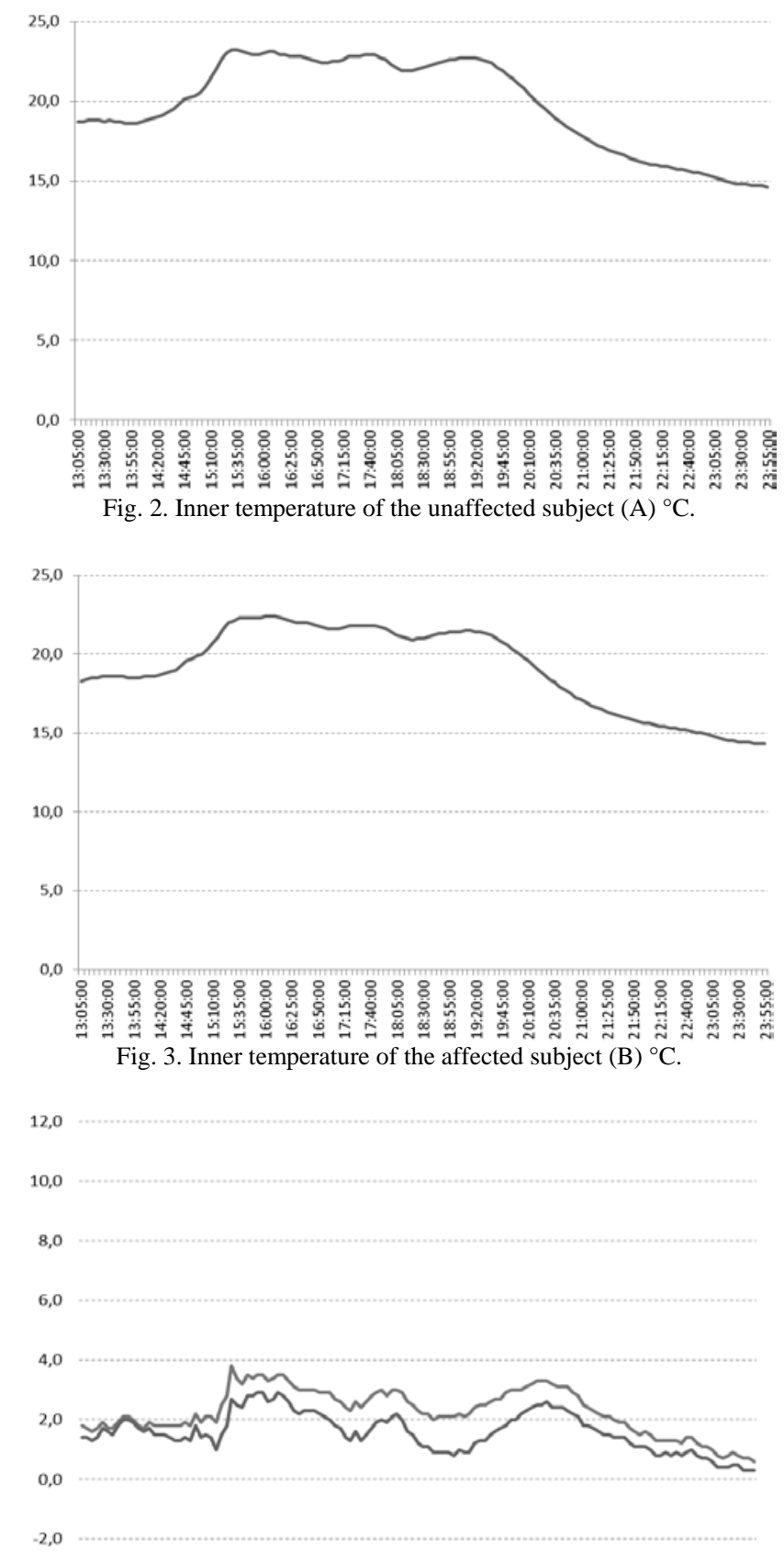

$-4,0$

Fig. 4. Inner temperature of the affected and unaffected subject (A), (B) with the subtraction of the exterior temperature - showing the solar gains of both subjects (A), (B) ${ }^{\circ} \mathrm{C}$, where the upper amplitude expresses the behavior of the inner environment of unaffected subject (A) and the lower amplitude the behavior of the inner environment of affected subject (B). 
The experiment runs on two subjects: Cargo container (A)-without the reflective layer applied on its inner shell, therefore "unaffected" - and the second subject Cargo container (B) - with applied reflective layer of material on its inner shell, therefore "affected". This way we have the opportunity of comprising the affected and unaffected subjects placed on the same grounds with the same exterior properties.

The testing ground was picked with the caution to exposition to sun shine, shade, air movement and distance from other heat absorbing/radiating objects. Before the main measuring started, the team conducted precise measuring of the sky light and the $360^{\circ}$ photographs of the sky were taken to prove that both tested subjects have the same external parameters. Both subjects are placed in the sufficient distance, so the subjects are not affecting each other in terms of heat radiation and shade.

On both subjects are measured:

1. Contact temperature of external shell - in direct contact with the exterior

2. Contact temperature of the inner shell - on the interior side of shell

3. Ambient temperature of the inner environment of subjects

4. Ambient humidity of the inner environment of subjects

5. External ambient temperature

6. External ambient humidity

All the measured data are collected for further survey and are constantly evaluated and compared. From the first measuring yield are presented graphs that shows the amplitude of the ambient temperatures in affected and unaffected subject and are compared with the ambient temperature of the exterior.

This comparison provides sufficient data to interim conclusions that the material has a thermal impact on the subject (B) in the terms of ambient inner temperature.

\section{CONCLUSION-EVAlUATION OF EXISTING RESEARCH, OUTLINING AdDITIONAL IDEAS FOR THE NEXT PHASE OF RESEARCH}

\section{A. Evaluation of the Current Research}

Plan for the year 2016 was the production of standardized samples using the investigated material ("Glass Bubbles" 3M (tm) glass microspheres, 3M (tm) Glass Bubbles source: http://solutions.3mcesko.cz). Specification of functional layers and best practice for manufacturing of standardized components with defined quality and repeatable manufacturing technology that can be used in laboratory tests.

When developing the samples team partnered with AZ TECH, Ltd., who is delegated by $3 \mathrm{M}$ as a converter of specific building materials. In cooperation with the said company was developed the specific manufacturing process.

In manufacturing first studied subjects was used input information and technology provided by AZ Tech, Ltd. which are, based on the input of research, further technically developed, and are gradually upgraded to meet the carrier agent and the binder used for the investigated material so it meet the best intent of the school laboratory research.

Standardized samples were made of and are now the subject of an experimental measuring of their thermal and -optical properties.

\section{B. Mid-Term Research Plan - Stabilizing Ideas for Next Phase}

An important stage in the research will be establishing cooperation with domestic manufacturers of glass and developing specialized methods to standardize the production of large samples for validation of the results of input measurements.

Along with initiating cooperation with manufacturers of structural glass will probably be extended to research collaboration with domestic glass production and introducing a new element of research from the fields of Arts, both with regard to design large glass surfaces and defining the technological limits of applicability of the material in large-scale.

Another possible cooperation in the field of architectural glass is possible use of the material in the exterior, the on-going testing of non-transparent materials is very promising and anticipated milestone in bringing this technology to the Czech market is the end of 2016. After the approval of material solutions for the design of the exterior is reasonable to assume establish cooperation in the areas of reconstruction and the refurbishment of existing large-area glazing.

\section{Experiment Conclusion}

The undergoing first phase of the field experiment on larger scale subjects proves the positive thermal impact on the inner environment of the tested subject. The material partially blocks the solar income through the thin walls of the cargo container and causes the lowering of the inner temperature gains.

The experiment also brings new research questions in terms of the thickness of the reflective layer - The reflective material on the inner shell of the container is, because the larger scale and the prismatic shape of the walls, applied manually and that does not guarantee the coherent layer of the material with the same exact thickness on all the area of application, so the solar gains are not equal between the sides of the container when it comes to position and direction of the sunrays - and the not sufficient scale of the experiment in terms of infiltration of external air - The container is not air sealed and therefore vulnerable to wind gust that affects the subject in two ways: cooling the external shell with very low thermal capacitance and also by infiltrating the exterior air into the interior of the subject.

This newly added research problems are dealt with in the second phase of the field experiment that includes the precise monitoring of the external parameters such as amount and vector of the solar radiation and also the windpower. The gained data from the subjects A and B will be processed only after the wind will be very low and the solar radiation will be sufficiently stable.

\section{ACKNOWLEDGMENT}

The authors wish to thank to Czech Technical University in Prague for technical support and cooperation, especially to 


\section{Z. Vyoralová; to AZ-TECH Ltd. for consultancy and material support.}

\section{REFERENCES}

[1] S. N. Patankar and Y. A. Kranov, "Hollow glass microsphere HDPE composites for low energy sustainability,” Mater. Sci. Eng. A, vol. 527, pp. 1361-1366, 2010.

[2] L. Prokopová and D. Bošová, "Architectural models for measurement of daylight factor," Energy Saving and Environmentally Friendly Technologies - Concepts of Sustainable Building, vol. 824, 2016.

[3] J. Zajíc, G. Kuncová, M. Bittner, T. Branyik, A. Solovyev, S. Šabata, and M. Pospíšilová, "Repetitive inductions of bioluminescence of pseudomonas putida TVA8 immobilised by adsorption on optical fibre," Chemical Papers., vol. 70, no.7, pp. 877-887, 2016.

[4] A. S. Geleila, M. M. Hallb, and J. E. Shelbyc, "Hollow glass microspheres for use in radiation shielding," Journal of Non-Crystalline Solids, New Functionality of Glasses — in Proc. 17th University Conference on Glass Science, vol. 352, issues 6-7, 2006, pp. 620-625.

[5] Glass Bubbles-3M (tm) Glass Microspheres, Prague, 2003.

František Novotný was born on March 20, 1987, in Prague of Czech Republic. He graduated at the Faculty of Architecture, Czech Technical University in Prague master degree in "Multifunctional housing complexes in city centre" in 2012.

Since 2014 he is a student of PhD programme in Faculty of Architecture CTU. Since 2015 he is a lecturer in technical equipment of buildings and utilities of Institute of Civil Engineering II, Faculty of Architecture CTU in Prague. His specialized fields are: renewable energy, smart grids and district utilities. In the meantime he is the junior project manager at Centre for Central European Architecture, Prague and the senior architect at MOBA Studio, Prague

Since $2015 \mathrm{Mr}$. Novotny is the leading scientific investigator of grant project on Czech Technical University in Prague, focused on special coating materials for glass structures "Application of innovative coating materials for glass structures".

Daniela Bošová was born on February 22, 1973, in Prague of Czech Republic. He graduated from Czech Technical University in Prague-faculty of civil engineering. He got the $\mathrm{PhD}$ degree of "Distribution of moisture in the perimeter wall with contact insulation in relation to the occurrence of facultatively pathogenic mildew" in 2004.

From 1999 until 2012 she worked like lecturer in building structure focused on building physics-thermal, lighting and acoustics of the Department of Building structures, Faculty of Civil Engineering CTU in Prague. Since 2013 she works as a lecturer in building physics and fire safety of buildings of Institute of Civil Engineering II, Faculty of Architecture CTU in Prague. Her specialized fields are: daylighting, insolation and fire safety of buildings.

Ms. Bosova is authorized engineer for building structures and a member of Society for Environmental Technology. She worked on the ten research projects and grants, she was the principal investigator of three grant projects (one of them was international grant). She is a supervisor for $\mathrm{PhD}$ students. In scientific work, she focused on building physic and daylighting. She has done a lot of publications and university textbooks, including: daylighting, tubular light guide, building physics.

Lenka Prokopová was born on May 27, 1985, in Klatovy of Czech Republic. He graduated from Czech Technical University in Prague - faculty of civil engineering. He got the $\mathrm{PhD}$ degree of "Design and verification of methodology of daylight using light guide" in 2015.

Since 2015 she has worked like lecturer in building physics and technical equipment of buildings of Institute of Civil Engineering II, Faculty of Architecture CTU in Prague. Her specialized fields are: daylighting, tubular light guide and technical equipment of buildings. She worked on the five research projects and grants (one of them was international grant)

Ms. Prokopova was the principal investigator of three grant projects. In scientific work, she focused on Building Physic and Daylighting. She has done a lot of publications and university textbook, including: daylighting, tubular light guide, building physics-thermal, lighting and acoustics. 